\title{
All-Coefficient Adaptive Control of Dual-Motor Driving Servo System
}

\author{
Haibo Zhao ${ }^{1,2}$, Chengguang Wang ${ }^{3}$ \\ ${ }^{1}$ Engineering Technology Research Center of Optoelectronic Appliance, Tongling University, Tongling Anhui, 244061, China \\ ${ }^{2}$ Department of Electrical Engineering, Tongling University, Tongling Anhui, 244061, China \\ ${ }^{3}$ Sichuan Institute of Aerospace System Engineering, Chengdu Sichuan, 610100, China
}

\begin{abstract}
Backlash nonlinearity and friction nonlinearity exist in dual-motor driving servo system, which reducing system response speed, steady accuracy and anti-interference ability. In order to diminish the adverse effects of backlash and friction nonlinearity to system, we proposed a new all-coefficient adaptive control method. Firstly, we introduced the dynamic model of backlash and friction nonlinearity respectively. Then on this basis, we established the characteristic model when backlash and friction nonlinearity coexist. We used recursive least square method for parameter estimation. Finally we designed the all-coefficient adaptive controller. On the basis of simplex allcoefficient adaptive controller, we designed a feedforward all-coefficient adaptive controller. The simulations of feedforward all-coefficient adaptive control and simplex all-coefficient adaptive control were compared. The results show that the former has quicker response speed, higher steady accuracy, stronger anti-interference performance and better robustness, which validating the efficacy of the proposed control strategy.
\end{abstract}

\section{Introduction}

There are many nonlinearities which influencing the dynamic performance and steady-state accuracy of dualmotor driving servo system, such as backlash nonlinearity, friction nonlinearity, deadzone nonlinearity and so on in which the most obvious influence on system are backlash and friction nonlinearity. Thus, backlash and friction nonlinearity have always been caused extensive concern of academic and engineering at home and abroad. In terms of modeling of dual-motor driving servo system, many scholars at home and abroad have studied it. In [1], the dynamic model of dual-motor driving servo system containing backlash nonlinearity was established and the dynamic structure diagram of system was given. In [2], the simulation model of AC driving system based on dual-motor mutual feedback was established and the model was based on adaptive full order flux observer and torque sensor. In [3], a new compensation technique for friction that utilizes a backstepping control structure and an adaptive estimation of the friction force based on an observer for the class of dual-motor driving system was presented. In [4], the control model of machine tool feeding system driven by dual-motor containing backlash nonlinearity was established, using speed difference feedback to eliminate the synchronous error of dualmotor. In $[5,6]$, the mechanical model and experimental simulation block diagram of cement kiln driving system were given. But the system model established in the above literature are all dynamic models, and also the backlash and friction nonlinearity exist alone, without considering the system model when backlash and friction nonlinearity coexist.

In the compensate control strategies, many scholars at home and abroad have also studied them. In [7], the parameter identification of nonlinear dynamic systems with input saturation and output backlash using threeblock cascade models was presented. Examples of threeblock cascade system identification illustrated the feasibility of proposed method. In [8], aiming at unknown dynamic friction parameters and unknown load characteristic, the adaptive friction compensation was designed for the servo system. Simulation and experiment results were shown to effectively inhibit the disadvantageous influence of friction. In [9], an adaptive controller was designed for a class of periodically timevarying systems with input backlash, when the periodical uncertainties can be parameterized. The sign function was replaced by the hyperbolic function to ensure the differentiability for the controller and to avoid the chattering. In [10], an iterative learning control method was presented for a class of time-varying nonlinear systems with input backlash. In [11], an adaptive fuzzy controller was designed in the case of unknown system parameters and load torque. In [12], a friction torque compensation controller without distal-end feedback was designed to ensure that the output of the system can effectively track the expected torque trajectory, and the effectiveness of the scheme was validated by experiments. But most of scholars at home and abroad have studied the control strategies when backlash and friction nonlinearity exist alone, few scholars have studied the compensate strategies when backlash and friction nonlinearity coexist. 
Taking a wide view of the development of backlash and friction nonlinearity in recent years, the main control algorithms are robust adaptive control, repetitive control, fuzzy control, sliding mode control, neural network control and so on. To some extent, the above proposed control methods have achieved good control effect, but still can't meet many problems in practical application, such as the dynamic tracking performance and control precision are not high, system parameter changes, etc. The all-coefficient adaptive control method has features of simple algorithms, strong robustness and so on, and has been successfully applied in actual industrial and aerospace control system, algorithm itself has a high maturity, but few scholars use it to control dual-motor driving servo system, so it is necessary to study it. Aiming at backlash and friction nonlinearity coexist, a new system model, namely characteristic model was established. We use all-coefficient adaptive control algorithm to control system and obtain the ideal control effect.

\section{Characteristic Model}

The so-called characteristic model is the modeling combining the dynamic characteristics of object, environmental characteristics, control performance requirements, not only depending on the accurate dynamic analysis of object. The features of characteristic model are as follows [13].

(1)Under the same control input, the object characteristic model is equivalent to the output of the actual object(namely in the dynamic process, system can keep within the allowable output error). In a steady state, the output are equal.

(2)The form and order of the characteristic model are mainly determined by the control performance requirements, in addition to the characteristics of the object.

(3)The form of characteristic model is simpler than the original object's dynamic equation, easy to design controller and easy to implement in engineering.

(4) The characteristic model is different from the reduced-order model of higher order system, which is to reduce the information of the higher order model into several characteristic parameters. In general, the characteristic model is described by the slowly time varying difference equation.

The characteristic model can be directly modeled by the object physical mechanism, and also can be derived from the original dynamic equation and the control performance requirements. In this paper, we adopt the latter method, firstly we establish the system dynamics model when backlash and friction nonlinearity coexist, then we design the system characteristic model on this basis.

\subsection{System Dynamic Model}

Fig. 1 is the schematic diagram of dual-motor driving servo system. $O_{0}$ is driven-subsystem, $O_{1}$ and $O_{2}$ are driving-subsystems.

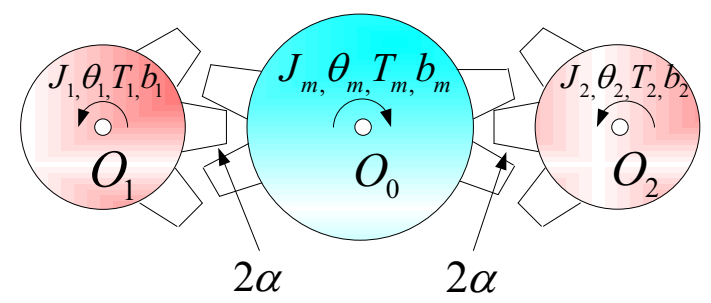

Figure 1. The schematic diagram of dual-motor driving servo system.

where $J_{1}, J_{2}$ are the rotational inertias of driving wheel. $J_{m}$ is the rotational inertia of driven wheel. $\theta_{1}, \theta_{2}$ are the rotation angles of driving wheel shaft. $\theta_{m}$ is the rotation angle of driven wheel shaft. $T_{1}, T_{2}$ are the input torques of driving wheel. $T_{m}$ is the load torque of driven wheel. $b_{1}, b_{2}$ are the viscous friction coefficients of driving wheel. $b_{m}$ is the viscous friction coefficient of driven wheel. $2 \alpha$ is the backlash.

Assumption 1 In the control process, drivensubsystem $O_{1}$ and $O_{2}$ contact with $O_{0}$ alternatively. It was guaranteed by exerting some bias torques to drivensubsystem from output of two driving-subsystems [14]. The bias torques was equal and opposite. Because of the influence of backlash nonlinearity [15], $\tau_{i}(t)(i=1,2)$ can be expressed as follows.

$$
\tau_{i}(t)=k f\left(z_{i}(t)\right)+c f\left(\dot{z}_{i}(t)\right)
$$

where, $k$ and $c$ are stiffness coefficient and damping coefficient, driving wheel and driven wheel, respectively. $f\left(z_{i}(t)\right)$ is the deadzone function and can be expressed as follows.

$$
f\left(z_{i}(t)\right)=\left\{\begin{array}{cc}
z_{i}(t)+\alpha & z<-\alpha \\
0 & -\alpha \leq z \leq \alpha \\
z_{i}(t)-\alpha & z>\alpha
\end{array}\right.
$$

where, $z_{i}(t)=\theta_{i}(t)-\theta_{m}(t) \quad$ (suppose the transmission ratio is 1$)$.

In dual-motor driving servo system, in addition to the influence of backlash nonlinearity, there is also the influence of friction nonlinearity. Let $\tau_{n}(t)(n=3,4)$ is the torque generated by friction in system (mainly on the driving wheel side). We use Stribeck friction model to model $\tau_{n}$.

$$
\tau_{n}(t)=\left[F_{c}+\left(F_{s}-F_{c}\right) e^{-\left(\dot{\theta}_{i}(t) / \dot{\theta}_{s}\right)^{\delta}}\right] \operatorname{sgn}\left[\dot{\theta}_{i}(t)\right]+b \dot{\theta}_{i}(t)
$$

where, $F_{c}, F_{s}, b, \dot{\theta}_{i}(t), \dot{\theta}_{s}$ are Coulomb friction, maximum static friction force, viscous friction coefficient, angular velocity of driving wheel and Stribeck velocity, respectively. $\dot{\theta}_{s}$ and $\delta$ are empirical constants. Usually $\dot{\theta}_{s}=0.05, \delta=2$.

The typical dynamic equation of dual-motor driving servo system is as follows. 


$$
\left\{\begin{array}{l}
U_{i}(t)=R_{i} I_{i}(t)+L_{i} \frac{d I_{i}(t)}{d t}+C_{i e} \dot{\theta}_{i}(t) \\
J_{i} \ddot{\theta}_{i}(t)+b_{i} \dot{\theta}_{i}(t)=T_{i}-\tau_{i}-\tau_{n} \\
J_{m} \ddot{\theta}_{m}(t)+b_{m} \dot{\theta}_{d}(t)=\tau_{1}+\tau_{2} \\
T_{i}=k_{d i} I_{i}(t) \\
\tau_{i}(t)=k f\left(z_{i}(t)\right)+c f\left(\dot{z}_{i}(t)\right) \\
f\left(z_{i}(t)\right)=\left\{\begin{array}{cc}
z_{i}(t)+\alpha & z<-\alpha \\
0 & -\alpha \leq z \leq \alpha \\
z_{i}(t)-\alpha & z>\alpha
\end{array}\right. \\
\tau_{n}(t)=\left[F_{c}+\left(F_{s}-F_{c}\right) e^{-\left(\dot{\theta}_{i}(t) / \dot{\theta}_{s}\right)^{\delta}}\right] \operatorname{sgn}\left[\dot{\theta}_{i}(t)\right]+b \dot{\theta}_{i}(t)
\end{array}\right.
$$

where, $U_{i}(t)$ is the input electromotive force of driving motor. $R_{i}$ and $I_{i}$ are the armature circuit resistance and armature current of driving motor, respectively. $L_{i}$ is the armature circuit inductance of driving motor. $C_{i e}$ is the back EMF coefficient of driving motor. $k_{d i}$ is the torque coefficient of driving motor.

\subsection{System Characteristic Model}

Suppose a nonlinear system is as follows:

$$
\dot{x}(t)=f\left(x, \dot{x}, \cdots, x^{(n)}, u, \dot{u}, \cdots, u^{(m)}\right)
$$

let

$$
\begin{aligned}
& x=x_{1}, \dot{x}=x_{2}, \ldots, x^{(n)}=x_{n+1} \\
& u=u_{1}, \dot{u}=u_{2}, \ldots, u^{(m)}=u_{m+1}
\end{aligned}
$$

then Eqs.(5) can be expressed as follows:

$$
\dot{x}_{1}(t)=f\left(x_{1}, \cdots, x_{n+1}, u_{1}, \cdots, u_{m+1}\right)
$$

The following assumptions are made for the nonlinear system:

Assumption 2 The system is single input and single output.

Assumption 3 The order of control variable $u(t)$ is 1 .

Assumption 4 If all the independent variables of $f(\cdot)$ are zero, then $f(\cdot)=0$.

Assumption 5 All the independent variables in $f(\cdot)$ are continuous and differentiable, and all the partial derivatives are bounded.

Assumption $6|f(x(t+\Delta t), u(t+\Delta t))|-|f(x(t), u(t))|$ $<M \Delta t$, where $M$ is a positive constant, $\Delta t$ is sampling period.

Assumption 7 All the variables $x_{i}$ and $u_{i}$ are bounded.

Theorem 1 If the controlled object can be described by nonlinear system Eqs.(5), and meet the above assumptions 2-5, under the condition of satisfying certain sampling period $\Delta t$, its characterisric model can be described by a second order time varying difference equation:

$x(k+1)=f_{1}(k) x(k)+f_{2}(k) x(k-1)+g_{0}(k) u(k)+g_{1}(k) u(k-1)(7)$

When the controlled object is stable, and meet assumptions 5-6, then:

(1)In the dynamic process, the output of the characteristic model is equivalent to the actual output under the condition of the same input $u$ (proper selection of sampling period $\Delta t$ can guarantee the output error within the allowable range). The output are equal in steady state.

(2)The coefficients of $f_{i}(k)$ and $g_{i}(k)$ in eqs.(7) are slowly time varying.

(3) Coefficient range can be determined in advance.

By the theorem 1, we can see that only when all the independent variables of the system are continuous diferentiable, the characteristic model can be designed. But in the eqs.(4), the deadzone function and the sign function are not differentiable, so they must be smoothed. We use a smooth, continuous and differentiable function in place of deadzone function[16]:

$$
f\left(z_{i}(t)\right)=z_{i}-4 \alpha\left(\frac{2}{1+e^{-r z_{i}}}-1\right)
$$

so,

$$
\tau_{i}(t)=k\left(z_{i}-4 \alpha\left(\frac{2}{1+e^{-r z_{i}}}-1\right)\right)+c \dot{z}_{i}\left(1-8 r \alpha \frac{e^{-r z_{i}}}{\left(1+e^{-r z_{i}}\right)^{2}}\right)
$$

We use a smooth friction model to replace the Stribeck friction model[17], namely the introduction of driven wheel speed amplification coefficient, and adopt the arc tangent function of the driven wheel speed replace the symbol function sgn in Stribeck friction model. Smooth friction model is shown as follows.

$$
\tau_{n}(t)=\left[F_{c}+\left(F_{s}-F_{c}\right) e^{-\left(\dot{\theta}_{i}(t) / \dot{\theta}_{s}\right)^{s}}\right] \frac{2}{\pi} \arctan \left(k_{b} \theta_{i}(t)\right)+b \dot{\theta}_{i}(t)
$$

where $k_{b}>0$ is the speed amplification coefficient of driven wheel.

After the smooth treatment of backlash and friction nonlinearity respectively, the dynamic equation of dualmotor driving servo system with backlash and friction nonlinearity coexist is as follows.

$$
\left\{\begin{array}{l}
U_{i}(t)=R_{i} I_{i}(t)+L_{i} \frac{d I_{i}(t)}{d t}+C_{i e} \dot{\theta}_{i}(t) \\
J_{i} \ddot{\theta}_{i}(t)+b_{i} \dot{\theta}_{i}(t)=k_{d i} I_{i}(t)-\tau_{i}-\tau_{n} \\
J_{m} \ddot{\theta}_{m}(t)+b_{m} \dot{\theta}_{d}(t)=\tau_{1}+\tau_{2} \\
\tau_{i}(t)=k\left(z_{i}-4 \alpha\left(\frac{2}{1+e^{-r z_{i}}}-1\right)\right)+c \dot{z}_{i}\left(1-8 r \alpha \frac{e^{-r z_{i}}}{\left(1+e^{-r z_{i}}\right)^{2}}\right) \\
\tau_{n}(t)=\left[F_{c}+\left(F_{s}-F_{c}\right) e^{\left.-\left(\dot{\theta}_{i}(t)\right) \dot{\theta}_{s}\right)^{\delta}}\right] \frac{2}{\pi} \arctan \left(k_{b} \theta_{i}(t)\right)+b \dot{\theta}_{i}(t)
\end{array}\right.
$$

let $x_{1}=\theta_{i}(t), x_{2}=\dot{\theta}_{i}(t), x_{3}=\theta_{m}(t), x_{4}=\dot{\theta}_{m}(t)$, $x_{5}=I_{i}(t)$, then the state equation of system is as follows.

$$
\left\{\begin{array}{l}
\dot{x}_{1}=x_{2} \\
\dot{x}_{2}=\frac{1}{J_{i}}\left(k_{d i} x_{1}-\tau_{i}-\tau_{n}-b_{i} x_{2}\right) \\
\dot{x}_{3}=x_{4} \\
\dot{x}_{4}=\frac{1}{J_{m}}\left(\tau_{i}-b_{i} x_{4}\right) \\
\dot{x}_{5}=\frac{1}{L_{i}}\left[U_{i}(t)-C_{i e} x_{2}-R x_{5}\right] \\
y=x_{3}
\end{array}\right.
$$

From theorem 1, we can get the characteristic model of system is as follows.

$\theta_{m}(k+1)=f_{1}(k) \theta_{m}(k)+f_{2}(k) \theta_{m}(k-1)+g_{0}(k) u(k)+g_{1}(k) u(k-1)$ 


$$
=\phi^{T}(k-1) \theta(k)
$$

where,

$$
\begin{gathered}
\phi(k-1)=\left[\begin{array}{llll}
\theta_{m}(k) & \theta_{m}(k-1) & u(k) & u(k-1)
\end{array}\right]^{T}, \\
\theta(k)=\left[\begin{array}{lllll}
f_{1}(k) & f_{2}(k) & g_{0}(k) & g_{1}(k)
\end{array}\right]^{T}
\end{gathered}
$$

The recursive least square method is used to estimate the parameters $f_{1}(k), f_{2}(k), g_{0}(k), g_{1}(k)$ (forgetting factor $\lambda=0.97$ ).

$$
\begin{gathered}
K(k)=\frac{P(k-1) \phi(k-1)}{\lambda+\phi^{T}(k-1) P(k-1) \phi(k-1)} \\
\hat{\theta}(k)=\hat{\theta}(k-1)+K(k)\left[y(k)-\phi^{T}(k-1) \hat{\theta}(k-1)\right] \\
P(k)=\frac{1}{\lambda}\left[I-K(k) \phi^{T}(k)\right] P(k-1)
\end{gathered}
$$

\section{Controller Design}

The main control object of all-coefficient adaptive control is the linear time-invariant system with unknown parameters or some nonlinear systems. Its main feature is to establish a mathematical model whose sum of all coefficients is equal to 1 , and to design the adaptive controller on the basis of determining parameters range. Because this algorithm has the characteristics of debugging less parameters, simple algorithm and strong robustness, it has been successfully applied in engineering practice. In this paper, aiming at the characteristic model of dual-motor driving servo system, we combined the golden section adaptive control and the logical integral control. The system control block diagram is as follows.

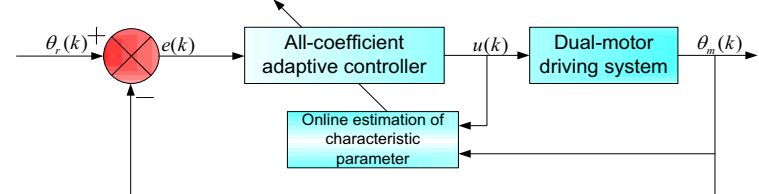

Figure 2. The block diagram of all-coefficient adaptive control. where, $u(k)$ is the output control variable of allcoefficient adaptive controller, its expressions is as follows.

$$
u(k)=u_{1}(k)+u_{i}(k)
$$

where, $u_{1}(k)$ is the golden section control variable, $u_{i}(k)$ is the logical integration control variable.

$u_{1}(k)=\frac{-1}{\hat{g}_{0}(k)+\eta}\left[l_{1} \hat{f}_{1}(k) e(k)+l_{2} \hat{f}_{2}(k) e(k-1)+\hat{g}_{1}(k) u_{1}(k-1)\right]$

where, $\hat{g}_{0}(k)$ and $\hat{g}_{1}(k)$ are estimated values of $g_{0}(k)$ and $g_{1}(k)$, respectively. $e(k)=\theta_{m}(k)-\theta_{r}(k)$.

Let $k_{i}^{+}=k_{i}^{-}, c=1$, then the logical integration control variable is as follows [18].

$$
u_{i}(k)=u_{i}(k-1)-k_{i}\left(\theta_{m}(k)-\theta_{r}(k)\right)
$$

and when reference input $\theta_{r}(k)-\theta_{r}(k-1)=0$, $u_{i}(k)=0$.

If the feedforward control is added in Fig. 2, then system block diagram based on the feedforward allcoefficient adaptive control is as follows.

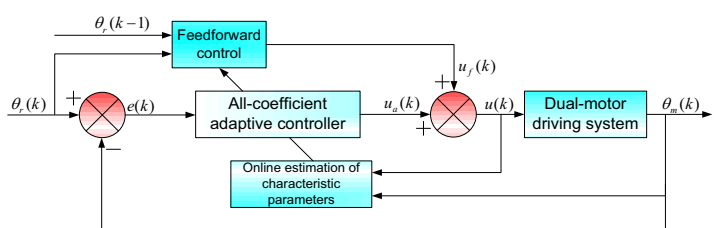

Figure 3. The block diagram of feedforward+allcoefficient adaptive control.

where, $u_{f}(k)=k_{f}\left(\theta_{r}(k)-\theta_{r}(k-1)\right)$ is the feedforward control variable, $k_{f}$ is the feedforward control coefficient, $u_{a}(k)$ is the output control variable of all-coefficient adaptive controller, the output control variable of feedforward all-coefficient adaptive controller is as follows.

$$
u(k)=u_{a}(k)+u_{f}(k)=u_{1}(k)+u_{i}(k)+u_{f}(k)
$$

\section{Simulation Experiment}

The physical parameters of system are shown in Table 1.

Table 1. The system physical parameters.

\begin{tabular}{|c|c|}
\hline$J_{1}, J_{2}$ & $0.185 \mathrm{~kg} \cdot \mathrm{m}^{2}$ \\
\hline$J_{m}$ & $0.028 \mathrm{~kg} \cdot \mathrm{m}^{2}$ \\
\hline$b_{1}, b_{2}$ & $1.2 \mathrm{~N} \cdot \mathrm{m} \cdot \mathrm{s} / \mathrm{rad}$ \\
\hline$b_{m}$ & $1.3 \mathrm{~N} \cdot \mathrm{m} \cdot \mathrm{s} / \mathrm{rad}$ \\
\hline$k$ & {$[560+2 \mathrm{sin}(\pi t)] \mathrm{N} \cdot \mathrm{m} / \mathrm{rad}$} \\
\hline$c$ & {$[0.15+0.01 \mathrm{sin}(\pi t)] \mathrm{N} \cdot \mathrm{m} / \mathrm{rad}$} \\
\hline$\alpha$ & $0.5 \mathrm{rad}$ \\
\hline$w$ & $50 \mathrm{~N} \cdot \mathrm{m}$ \\
\hline
\end{tabular}

During the simulation, we compare the output response based on all-coefficient adaptive control (ACAC), feedforward all-coefficient adaptive control (FACAC), respectively. The parameters of all-coefficient adaptive controller $\eta=1.8 \times 10^{-4}, k_{i}=85$. The parameter of feedforward controller is $k_{f}=115$.

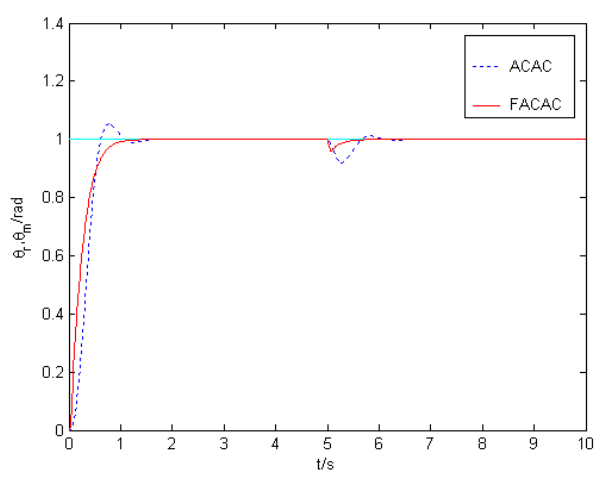

Figure 4. System tracking unit step response. 


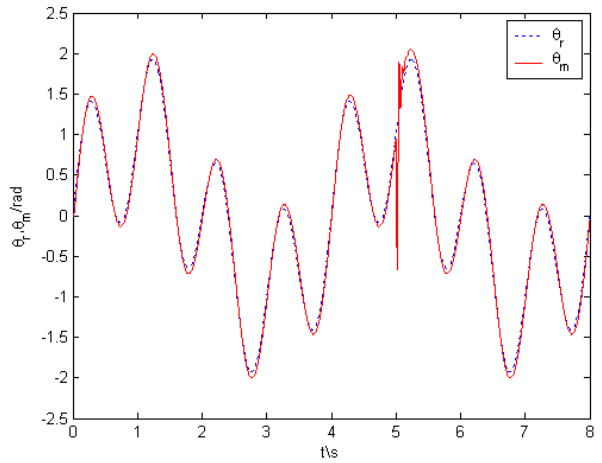

Figure 5. System tracking input response(ACAC).

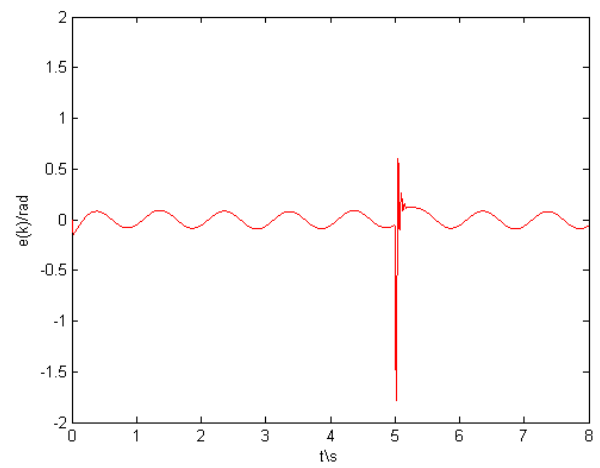

Figure 6. Tracking error curve(ACAC).

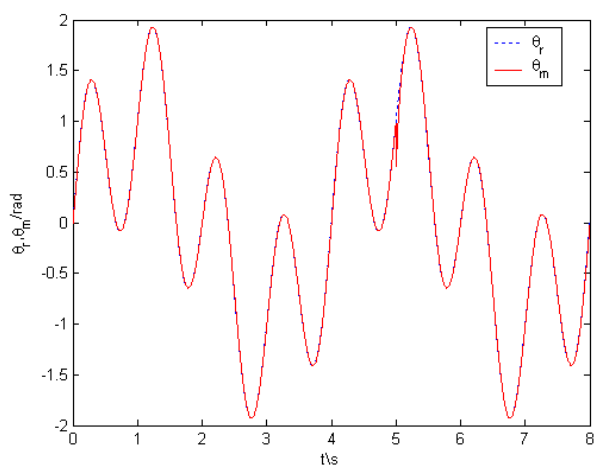

Figure 7. System tracking input response(FACAC).

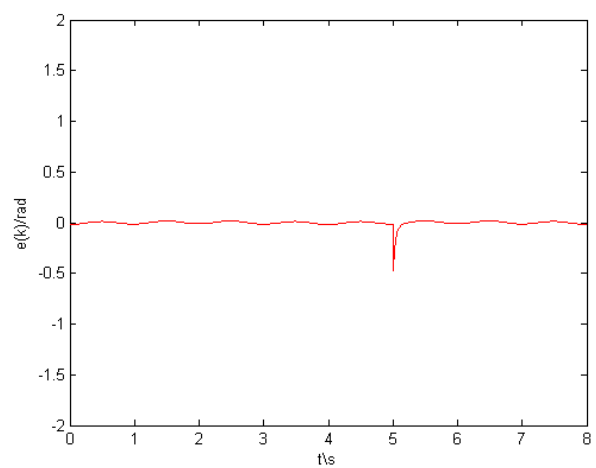

Figure 8. Tracking error curve (FACAC).

Fig. 4 is the output response of tracking unit step signal, we impose $5 \mathrm{Nm}$ disturbance on system at $5 \mathrm{~s}$; Fig. 5 and Fig. 7 are the output response of tracking input $\theta_{r}(t)=\sin (2 \pi t)+\sin (0.5 \pi t)$, their control strategies are ACAC and FACAC, respectively, and we impose $8 \mathrm{Nm}$ disturbance on system at 5s; Fig. 6 and Fig. 8 are tracking error curves using ACAC and FACAC, respectively.
From Fig. 4 to Fig. 8, we can see that system has faster response speed, smaller tracking error, stronger antijamming capability and stronger robustness when FACAC method is used.

\section{Conclusion}

Aiming at the dual-motor driving servo system when backlash and friction nonlinearity coexist, the system characteristic model was established, the controllers based on all-coefficient adaptive control and feedforward all-coefficient adaptive control were designed, respectively. The simulation results show the superiority of the feedforward all-coefficient adaptive control method, which provides a reference for the further research of dual-motor driving servo system.

\section{Acknowledgment}

This work was supported by National Natural Science Foundation of China(No.61074023), Nature Science Foundation in Anhui Province of China(No.1508085MF130), Natural Science Research Key Project of Universities in Anhui Province of China (No.KJ2015A297), the Engineering Technology Research Center of Optoelectronic Appliance in Anhui Province of China, Sichuan Institute of Aerospace System Engineering in Sichuan Province of China.

The authors confirm that this article content has no conflicts of interest.

\section{References}

1. L. F. Sun, Research on motion characteristics and anti-backlash control of dual-motor system, Northeastern University, June 2012.

2. H. Z. Zhu, W. X. Song, Y. Dong, Modeling and simulation of coaxial connected dual motor drive system, Motor Contr. appl. 40(2) (2013) 23-27.

3. Y. Zhang, X. M. Ren, Adaptive backstepping control of dual-motor driving servo systems with friction, IHMSC 2014, 1, 214-217.

4. H. P. Ren, B. He, Anti-backlash control of machine tool feed system driven by dual-motors, Electr. Mach. Contr. 18(3) (2014) 60-66

5. I. X. Bogiatzidis, E. D. Mitronikas, A. N. Safaces, Investigation of backlash phenomena appearing in a twin AC cement kiln drive, Electrical Machines (ICEM), 2010, 1-6.

6. I. X. Bagiatzidis, A. N. Safacas, Vibration analysis and backlash identification of a twin AC drive for a cement kiln, Power Electronics, Machines and Drives (PEMD2010), 2010, 1-6.

7. Vrs, Jozef, Identification of nonlinear dynamic systems with input saturation and output backlash using three-block cascade models, J. Franklin Inst. 351(12) (2014) 5455-5466.

8. Y .F. Wu, D. W. Ma, J. Y. Yao, G. G. L, Nonlinear adaptive dynamic friction compensation for 
mechatronic servo systems, J. Mech. Eng. 49(6) (2014) 89-94.

9. S. Zhu, M. X. Sun, X. X. He, Adaptive control of a class of periodically time-varying nonlinearsystems with input backlash" Contr. Theor. Appl. 29(4) (2012) 535-538.

10. S. Zhu, M. X. Sun, X. X. He, Iterative learning control of a class of nonlinear systems with input backlash, Acta Automat. Sin. 37(8) (2011) 10141017.

11. R. H. Du, Y. F. Wu, W. Chen, Q. W. Chen, Adaptive fuzzy control for the servo system with LuGre friction" Control and Decision, 28(8) (2013) 12531256.

12. Q. S. Wu, X. S. Wang, F. P. Du, L. Chen, Torque transmission characteristics and friction compensation for a tendon-sheath actuation system in pull-pull configuration" J. Mech. Eng. 51(5) (2015) 22-29.

13. H. X. Wu, J. Hu, Y. C. Xie, Intelligent adaptive control based on characteristic model, China Science and Technology Publishing House, 2009.

14. H. B. Zhao, Study on control and simulation of dualmotor driving servo system, Compu. Simul. 29(3) (2012) 262-265.

15. G. TAO, X. MA, Y. LING, Optimal and nonlinear control of systems with sandwiched backlash, Automatica, 37(2) (2001) 165-176.

16. R. MEZOUKI, J. A. DAVILA, L. FRIDMAN, Backlash phenomenon observation and identification in electromechanical systems, Control Eng. Pract. 15(4) (2007) 447-457.

17. Y. Y. Li, Design and experimental research of servo system based on characteristic model, Nanjing University of Science and Technology, March, 2014.

18. F. Q. Huang, Study of characteristic model base on multi-motor driving servo system, Nanjing University of Science and Technology, March, 2013. 